\title{
El aburrimiento en estudiantes universitarios
}

\author{
Julio Torales, Iván Barrios
}

Extensa bibliografía ha señalado la relación existente entre el bienestar psicológico y diversas variables [1] de la vida de los estudiantes universitarios, incluidos los de medicina, como ansiedad, estrategias de afrontamiento, autoestima, autoeficacia, salud física, salud mental, patrones de personalidad, desarrollo económico y estilo de vida, asertividad y rendimiento académico. Asimismo, los investigadores han encontrado una fuerte correlación positiva entre el bienestar psicológico y un mejor rendimiento académico de los estudiantes universitarios [1].

Uno de los componentes más importantes del estudio del bienestar psicológico lo constituye el análisis del aburrimiento. Está demostrado que el aburrimiento contribuye negativamente al rendimiento de los estudiantes en las universidades. No obstante, el estudio formal del aburrimiento en la educación superior sigue siendo un campo relativamente poco desarrollado [2].

El aburrimiento se refiere a un estado psíquico de 'comodidad sin esfuerzo' durante el cual, paradójicamente, coexisten el deseo de disfrutar de la vida y sentir placer, pero sin estar en conexión con la voluntad, la concentración, la disciplina o la determinación de hacer algo para lograrlo. Estudios realizados sobre el bienestar psicológico en países como Paraguay han evidenciado un predominio de la condición de aburrimiento en la calidad de la experiencia subjetiva de estudiantes de medicina [1].

El aburrimiento es una emoción desagradable y desactivadora de la conducta $[3,4]$ que provoca en el estudiante la sensación de que el tiempo no transcurre, por lo que desea 'escapar' de esa situación. El aburrimiento se caracteriza por posturas o gestos que denotan falta de ilusión, de interés y de valoración de la actividad. Es reconocido que el aburrimiento obstaculiza el aprendizaje: los estudiantes más aburridos reportan menos horas de estudio y calificaciones más bajas que aquellos que no lo están [2]. Asimismo, algunos estudios lo relacionan con el consumo de drogas y la delincuencia juvenil, por lo que no podemos dejarlo pasar desapercibido con el objetivo de mejorar el proceso de enseñanzaaprendizaje.

Consideramos que la condición de aburrimiento es generada muchas veces por el ambiente y la falta de motivación que las universidades brindan a sus estudiantes. Si bien no es adecuado generalizar y afirmar que el aburrimiento es la única condición que puede afectar al rendimiento académico de los universitarios, es innegable resaltar la necesidad de asegurar un entorno que otorgue un buen nivel de calidad de vida a estos jóvenes y que proteja tanto su salud física como su mental [5]. Esto es vital para apoyar un adecuado desarrollo de su capacidad de aprendizaje [1].

Así, un contexto que no motiva a los estudiantes universitarios podría, a largo plazo, dificultar su cognición y afectos, conduciendo a la generación de una personalidad rígida e instalando en ellos cuadros ansiosos o depresivos que interfieran en su proceso educativo [5]. Esto último podría producir que las formas de estudiar y aprender de los universitarios se vuelvan cada vez más inflexibles, lo que resultaría en la utilización de técnicas y hábitos de estudio no siempre eficaces para el tipo de asignatura y su contexto, lo cual influirá en su rendimiento, satisfacción y emociones.

\section{Bibliografía}

1. Torales J, Troche Z, Ibarrola E, Micó G, Barrios I, Bolla L, et al. Subjective psychological wellbeing in medical students: a pilot study from Paraguay. International Journal of Culture and Mental Health 2016; 9: 373-80.

2. Sharp JG, Hemmings B, Kay R, Murphy B, Elliott S. Academic boredom among students in higher education: a mixed-methods exploration of characteristics, contributors and consequences. Journal of Further and Higher Education. Forthcoming 2017; doi: 10.1080/0309877X.2016.1159292.

3. Acee T, Kim H, Kim H, Kim J, Chu H, Kim M. Academic boredom in under-and over-challenging situations. Contemp Educ Psychol 2010; 35: 17-27.

4. Nett U, Goetz T, Daniels L. What to do when feeling bored? Students' strategies for coping with boredom. Learning and Individual Differences 2010; 20: 626-38.

5. Torales J, Girala N, Moreno M, Arce A, Trinidad S, Estigarribia E, et al. Depresión y ansiedad en estudiantes de medicina de la Universidad Nacional de Asunción. Revista Paraguaya de Psiquiatría 2013; 1: 12-28.
Dirección de Investigaciones; Facultad de Ciencias Médicas (J. Torales). Estudiante de Matemática Estadística y de Medicina (I. Barrios). Universidad Nacional de Asunción. San Lorenzo, Paraguay.

Correspondencia: Prof. Dr. Julio Torales. Dirección de Investigaciones. Facultad de Ciencias Médicas. Universidad Nacional de Asunción. Campus Universitario de la UNA. San Lorenzo, Paraguay.

E-mail: jtorales@med.una.py (c) 2017 FEM 